\title{
Systematic Analysis of Current Decay Time during Disruption in HYBTOK-II Tokamak
}

\author{
Masaaki OKAMOTO, Noriyasu $\mathrm{OHNO}^{1)}$ and Shuichi TAKAMURA ${ }^{2)}$ \\ Graduate School of Engineering, Nagoya University, Nagoya 464-8603, Japan \\ ${ }^{1)}$ EcoTopia Science Institute, Nagoya University, Nagoya 464-8603, Japan \\ ${ }^{2)}$ Faculty of Engineering, Aichi Institute of Technology, Toyota 470-0392, Japan
}

(Received 24 October 2007 / Accepted 24 November 2007)

\begin{abstract}
In order to estimate the electromagnetic force acting on vessel components during tokamak disruptions, an accurate prediction of the plasma current decay time is necessary. We have verified a current decay model based on a simple series circuit with a plasma resistance and an inductance. The circuit is employed for establishment of a plasma current decay time database using disruptive discharges in a small tokamak HYBTOK-II. An increase in the decay rate of the plasma current during the current quench phase was observed in experiments associated with an increase in the plasma resistance. This experimental result is consistent with the prediction of the model.
\end{abstract}

(C) 2008 The Japan Society of Plasma Science and Nuclear Fusion Research

Keywords: disruption, current quench, current decay rate, small tokamak

DOI: $10.1585 /$ pfr.3.006

\section{Introduction}

The magnitude of damages to tokamak devices during disruptions must be estimated accurately in order to design tokamak fusion reactors. These disruptions are accompanied by an intense heat load on the divertor during thermal quench (TQ), and large electromagnetic (EM) forces on the vacuum vessel and in-vessel components because of eddy and halo currents induced during current quench (CQ). The eddy and halo currents are induced by the time variation of the plasma current and a vertical displacement event (VDE) [1], respectively. The EM forces, which are generated by the interaction between these currents and magnetic fields, could be large enough to mechanically break the in-vessel components [2]. In order to estimate the EM force generated by the eddy current, an accurate prediction of the plasma current decay time is crucial. In a recent study, we used a current decay model based on a simple series circuit of the plasma resistance $R_{\mathrm{p}}$ and inductance $L_{\mathrm{p}}$ to analyze the current decay time $\tau$ where $\tau_{\text {model }}$ can be described by $L_{\mathrm{p}} / R_{\mathrm{p}}$. The database for ITER (International Thermonuclear Experimental Reactor) [3] is established by using the current decay time $\tau$ normalized by the plasma cross-sectional area $S$. However, a few problems have been encountered; the data of the normalized $\tau$ exhibit large scattering among different tokamaks and operational shots. Therefore, it is strongly recommended that the validity of the current decay model be experimentally evaluated. In addition, this model must demonstrate that the normalized $\tau_{\text {model }}$ strongly depends on the plasma resistivity during CQ. In other words, it is important to accurately evaluate the electron temperature and effective charge during CQ. There have been only a few measurements of the electron temperature during $\mathrm{CQ}$ in large and middle size tokamaks as it is difficult to perform measurements in these tokamaks because $\tau$ is very short, and the electron temperature is too low for the conventional diagnostic system in these devices to be measured during CQ. Electrostatic probes can measure the electron temperature during $C Q$. However, it is difficult to use the electrostatic probes in very hot plasma because the probe tips get severely damaged because of the enormous heat load during the disruptions. Therefore, the electron temperature is calculated using a numerical model, which is based on a power balance equation between joule heating and radiation loss because of impurity gases [4,5].

On the other hand, probe measurements in the plasma can be performed at disruptive discharges in small tokamaks. Current density profiles and mode structures of MHD (Magnetohydrodynamics) instabilities at disruptive discharges have been reported by the magnetic probe measurement in the following devices: TOSCA [6, 7], LT-3 [8,9], TBR-1 [10] and TORTUS [11]. However, the current decay time $\tau$ and electron temperature during CQ have not been reported for small tokamaks.

In this paper, the time evolution of the electron temperature during $\mathrm{CQ}$ is measured using a triple probe inserted into the small tokamak HYBTOK-II [12] and plasma resistivity is estimated by the classical Spitzer formula [13]. Simultaneously, the plasma internal inductance is estimated from the poloidal magnetic field, measured with the internal magnetic probe. This work aims to comprehensively verify the current decay model for establishment of the current decay time database using the obtained experimental data. 


\section{Model of Current Decay Time}

If tokamak plasma is assumed to be represented by a simple series circuit consisting of resistance $R_{\mathrm{p}}$ and inductance $L_{\mathrm{p}}$, loop voltage $V_{\text {loop }}$ is expressed as

$$
V_{\text {loop }}=\frac{\mathrm{d}}{\mathrm{d} t}\left(L_{\mathrm{p}} I_{\mathrm{p}}\right)+R_{\mathrm{p}} I_{\mathrm{p}}
$$

where $I_{\mathrm{p}}$ is the plasma current. If $R_{\mathrm{p}}$ and $L_{\mathrm{p}}$ are constant in time, and the absolute value of the right-hand side of Eq. (1) is much larger than the absolute value of $V_{\text {loop }}$, the temporal evolution of $I_{\mathrm{p}}$ can be expressed by the following equation:

$$
I_{\mathrm{p}}=I_{\mathrm{p} 0} \exp \left(-t / \tau_{\text {model }}\right),
$$

where $\tau_{\text {model }}=L_{\mathrm{p}} / R_{\mathrm{p}}$ is the time constant of $I_{\mathrm{p}}$ decay, and $I_{\mathrm{p} 0}$ is the plasma current before CQ. Equation (2) is valid when the current decay time is very short and plasma resistance is sufficiently large. When the current decay time can be approximated by $\tau_{\text {model }}=L_{\mathrm{p}} / R_{\mathrm{p}}$, the normalized current decay time $\tau_{\text {model }} / S$ can be expressed as

$$
\frac{\tau_{\text {model }}}{S}=\frac{L_{\mathrm{p}} / 2 \pi R_{0}}{\eta_{\mathrm{p}}}
$$

where $R_{0}$ is the plasma major radius, $\eta_{\mathrm{p}}$ is the plasma resistivity, and $\eta_{\mathrm{p}}=R_{\mathrm{p}} S / 2 \pi R_{0} . \tau_{\text {model }} / S$ has little dependence on the device size, because $L_{\mathrm{p}}$ is approximately proportional to $R_{0}$, and has a strong dependence on $\eta_{\mathrm{p}}$, which is primarily determined by the electron temperature $T_{\mathrm{e}}$ and effective charge $Z_{\text {eff }}$ in the classical Spitzer formula [13]. Thus, the database for ITER prediction is established in terms of the normalized current decay time $\tau / S$ [3]. However, $R_{\mathrm{p}}$ and $L_{\mathrm{p}}$ generally change in time, and occasionally the absolute values of the first and second terms in the right-hand side of Eq. (1) have a similar order of magnitude as $V_{\text {loop }}$. In this case, Eq. (3) cannot be valid. Therefore, to verify the current decay model, it is necessary to measure $V_{\text {loop }}, L_{\mathrm{p}}$, and $R_{\mathrm{p}}$ in time during CQ experimentally.

\section{Waveform of Disruptive Discharge in HYBTOK-II Tokamak}

HYBTOK-II is a small standard tokomak with a circular cross-section of limiter configuration. The major radius $R_{0}$ is $40 \mathrm{~cm}$, the minor radius of vacuum vessel $a$, and the limiter radius $a_{1}$ are 12.8 and $11 \mathrm{~cm}$, respectively [12]. Figure 1 shows a typical waveform of a disruptive discharge in the HYBTOK-II. Zero in time corresponds to the initiating time of CQ. Disruption was driven by ramping up $I_{\mathrm{p}}$ to reduce the plasma surface safety factor $q_{\mathrm{a}}\left(=a B_{\mathrm{t}} / R B_{\theta}\right)$, where $B_{\mathrm{t}}$ and $B_{\theta}$ are the toroidal and poloidal magnetic field strengths, respectively $[14,15]$. The typical parameters just before CQ are as follows: plasma minor radius is approximately $9.5 \sim 10.5 \mathrm{~cm}, q_{\mathrm{a}} \sim 3, I_{\mathrm{p}}=10-11 \mathrm{kA}$, and $B_{\mathrm{t}} \sim 0.25 \mathrm{~T}$. It is found that the waveform of CQ consists of two phases of slow and fast current decays in

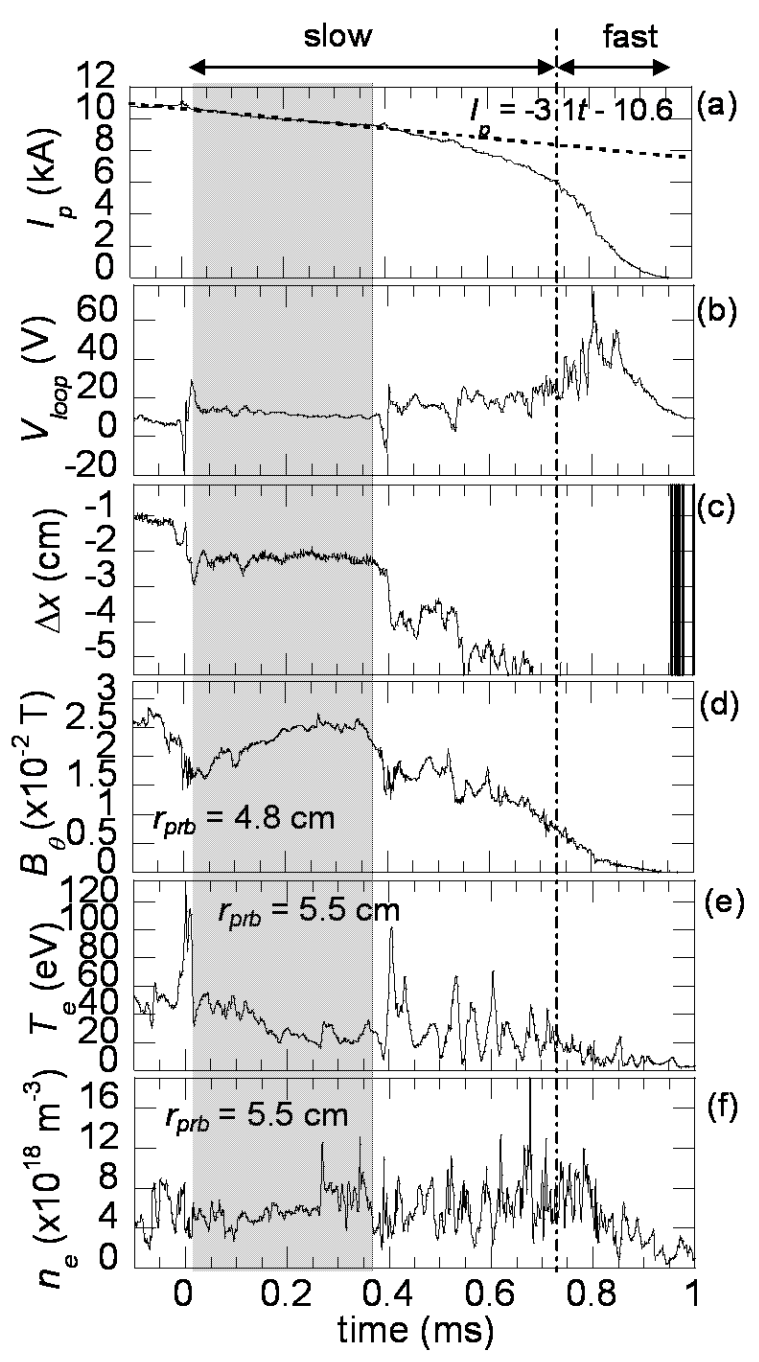

Fig. 1 Typical disruption waveform in HYBTOK-II $\left(B_{\mathrm{t}} \sim\right.$ $0.25 \mathrm{~T}$ ). Temporal evolutions of (a) plasma current $I_{\mathrm{p}}$, (b) plasma loop voltage $V_{\text {loop }}$, (c) horizontal position of plasma center $\Delta x$, (d) poloidal magnetic field $B_{\theta}$ at $r_{\mathrm{prb}}=4.8 \mathrm{~cm}$ and (e), (f) electron temperature $T_{\mathrm{e}}$ and density $n_{\mathrm{e}}$ at $r_{\text {prb }}=5.5 \mathrm{~cm}$. The negative value of the plasma horizontal position corresponds to the high field side and $\Delta x=0$ is the center of the vacuum vessel.

the HYBTOK-II disruptions as shown in Fig. 1 (a). In this paper, the current decay model is evaluated using a decay rate of $I_{\mathrm{p}}$ during the slow decay phase, because the plasma inductance and cross-sectional area can be experimentally obtained from the poloidal magnetic fields inside the plasma (Fig. 1(d)) and the horizontal position of the plasma center from the center of vacuum vessel (Fig. 1 (c)), respectively. The electron temperature $T_{\mathrm{e}}$ is measured by a triple probe located at $r_{\mathrm{prb}}=5,5.5$, and $6 \mathrm{~cm}$, and poloidal magnetic field $B_{\theta}$ is measured by a magnetic probe at $r_{\text {prb }}=4.3,4.8$, and $5.3 \mathrm{~cm}$, where $r_{\text {prb }}$ is the distance between the central position of the vacuum vessel and each probe position. The probe and magnetic probe are inserted vertically along the minor radius from the bottom of the 
vacuum vessel.

The time derivative of $I_{\mathrm{p}}$, denoted by $\Delta I_{\mathrm{p}} / \Delta t$, is determined by linear fitting during the initial phase of the slow decay, represented by a dashed line in Fig. 1 (a); here, $\Delta I_{\mathrm{p}} / \Delta t$ becomes $-3.1 \mathrm{kA} / \mathrm{ms}$. The plasma inductance is evaluated from time-averaged values of $B_{\theta}$ and $I_{\mathrm{p}}$ over the hatched region in Fig. 1 on the basis of Ampere's Law with an assumption of the following current density profile [16]:

$$
j(r)=j_{0}\left\{1-\left(r / a_{\mathrm{p}}\right)^{2}\right\}^{v} .
$$

Substituting Eq. (4) into the differential form of Ampere's law, $\mu_{0} j=(1 / r) \mathrm{d}\left(r B_{\theta}\right) / \mathrm{d} r, B_{\theta}$ can be expressed by the following equation:

$$
B_{\theta}(r)=\frac{\mu_{0} j_{0} a_{\mathrm{p}}^{2}}{2(v+1)} \frac{1-\left\{1-\left(r / a_{\mathrm{p}}\right)^{2}\right\}^{v+1}}{r},
$$

where $a_{\mathrm{p}}=a_{1}+\Delta x . \Delta x$ is the displacement of the center of the plasma column shown in Fig. 1 (c), and $a_{\mathrm{p}}$ is a plasma minor radius. The value of $B_{\theta}\left(a_{\mathrm{p}}\right)$ can be calculated from the integral form of Ampere's Law, $B_{\theta}\left(a_{\mathrm{p}}\right)=$ $\mu_{0} I_{\mathrm{p}} / 2 \pi a_{\mathrm{p}}$, using the measured value of $I_{\mathrm{p}}$. Peaking factor $v$ in Eq. (5) can be determined by $B_{\theta}\left(a_{\mathrm{p}}\right)$ and $B_{\theta}\left(r_{\mathrm{p}}\right)$, where $r_{\mathrm{p}}=\sqrt{\Delta x^{2}+r_{\mathrm{prb}}^{2}}$. Therefore, the plasma inductance $L_{\mathrm{p}}$ can be calculated from

$$
\begin{aligned}
& l_{\mathrm{i}}=\frac{\overline{B_{\theta}(r)^{2}}}{B_{\theta}\left(a_{\mathrm{p}}\right)^{2}}=\frac{2 \int_{0}^{a_{\mathrm{p}}} B_{\theta}(r)^{2} r \mathrm{~d} r}{a_{\mathrm{p}}^{2} B_{\theta}\left(a_{\mathrm{p}}\right)^{2}}, \\
& L_{\mathrm{p}}=\mu_{0} R_{0}\left(\frac{l_{\mathrm{i}}}{2}+\ln \frac{8 R_{0}}{a_{\mathrm{p}}}-2\right) .
\end{aligned}
$$

Time-averaged $I_{\mathrm{p}}$ and $B_{\theta}$ over the hatched region in Fig. 1 give $\langle v\rangle=1.56,\left\langle l_{\mathrm{i}}\right\rangle=1.09$, and $\left\langle L_{\mathrm{p}}\right\rangle=1.0 \mu \mathrm{H}$, where \langle\rangle indicates that the values are time-averaged. Therefore, $\left|\left\langle L_{\mathrm{p}}\right\rangle \Delta I_{\mathrm{p}} / \Delta t\right|$ can be estimated to be $3.1 \mathrm{~V}$, which is in the same order of magnitude as the time-averaged $V_{\text {loop }}$ of 11.7 V. It is found that Eqs. (2) and (3) cannot be used during the slow decay phase in Fig. 1 (a), therefore, we need to verify Eq. (1) directly.

\section{Verification of Current Decay Model Based on a Simple Series Circuit}

We have analyzed 115 disruptive shots. Figure 2 shows a histogram of $\left\langle T_{\mathrm{e}}\right\rangle$ taken among these 115 disruptive shots. $\left\langle T_{\mathrm{e}}\right\rangle$ indicates the time-averaged value of $T_{\mathrm{e}}$ during the slow decay phase. $T_{\mathrm{e}}$ is measured at different radial positions, $r_{\mathrm{prb}}=5,5.5$, and $6 \mathrm{~cm}$. The $\left\langle T_{\mathrm{e}}\right\rangle$ has little dependence on the radial position because the radial profile of $T_{\mathrm{e}}$ is flat. However, a large variation of $\left\langle T_{\mathrm{e}}\right\rangle$ among the disruptive shots appears even at the same radial position. The large variation could be caused by a difference in the particle recycling rate because of inward shift of the plasma column. We can verify Eq. (1) at range of $\left\langle T_{\mathrm{e}}\right\rangle$ from about 30 to $50 \mathrm{eV}$.

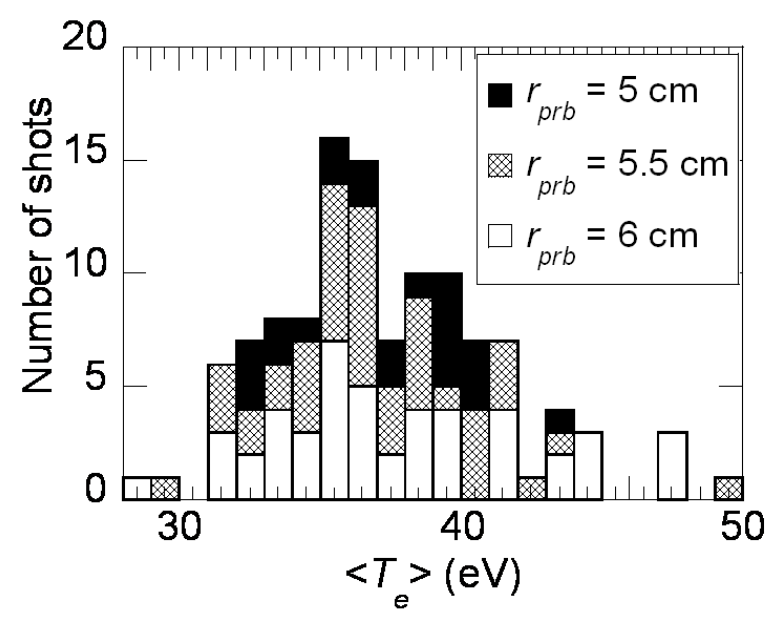

Fig. 2 Histogram of time-averaged electron temperature $\left\langle T_{\mathrm{e}}\right\rangle$ during the slow decay phase.

In order to evaluate the validity of Eq. (1), we need to estimate the plasma resistivity in the hatched region during slow decay phase of CQ. The plasma resistivity can be calculated using the classical Spitzer formula [13],

$$
\eta_{\mathrm{p}}=1.65 \times 10^{-9} Z_{\mathrm{eff}} T_{\mathrm{e}}^{-3 / 2} \ln \Lambda, \quad T_{\mathrm{e}} \text { in } \mathrm{keV}
$$

where $Z_{\text {eff }}$ and $\ln \Lambda$ are the effective charge and Coulomb logarithm, respectively. There are two methods to calculate the time-averaged plasma resistivity $\left\langle\eta_{\mathrm{p}}\right\rangle$ over the hatched region. A simple method is to calculate $\left\langle\eta_{\mathrm{p}}\right\rangle$ by substituting $\left\langle T_{\mathrm{e}}\right\rangle$ into Eq. (8), referred to as $\eta_{\mathrm{p}}\left(\left\langle T_{\mathrm{e}}\right\rangle\right)$. In another method, after calculating the time evolution of $\eta_{\mathrm{p}}(t)$ by substituting $T_{\mathrm{e}}$ in Eq. (8) by the $T_{\mathrm{e}}$ value shown in Fig. $1(\mathrm{e}),\left\langle\eta_{\mathrm{p}}\right\rangle$ is calculated by averaging $\eta_{\mathrm{p}}(t)$ over the hatched region in Fig. 1, and $\left\langle\eta_{\mathrm{p}}\right\rangle$ is denoted by $\left\langle\eta_{\mathrm{p}}\left(T_{\mathrm{e}}\right)\right\rangle$. In general, $\left\langle\eta_{\mathrm{p}}\left(T_{\mathrm{e}}\right)\right\rangle$ becomes larger than $\eta_{\mathrm{p}}\left(\left\langle T_{\mathrm{e}}\right\rangle\right)$ because of the nonlinear dependence of $\eta_{\mathrm{p}} \propto T_{\mathrm{e}}^{-3 / 2}$. Figure 3 shows the comparison between $\eta_{\mathrm{p}}\left(\left\langle T_{\mathrm{e}}\right\rangle\right)$ and $\left\langle\eta_{\mathrm{p}}\left(T_{\mathrm{e}}\right)\right\rangle$ at $Z_{\mathrm{eff}}=1$. It is found that $\left\langle\eta_{\mathrm{p}}\left(T_{\mathrm{e}}\right)\right\rangle$, calculated by the temporal evolution of $T_{\mathrm{e}}$, becomes larger than $\eta_{\mathrm{p}}\left(\left\langle T_{\mathrm{e}}\right\rangle\right)$ calculated by time-averaged value of $T_{\mathrm{e}}$. This indicates that the measurement of electron temperature with good time resolution is important for estimating the precise plasma resistance to evaluate the current decay model. We will use the values of $\left\langle\eta_{\mathrm{p}}\left(T_{\mathrm{e}}\right)\right\rangle$ hereafter.

If $R_{\mathrm{p}}$ and $L_{\mathrm{p}}$ are constant, using Eq. (1), the rate of $I_{\mathrm{p}}$ decay can be expressed as

$$
\frac{\mathrm{d} I_{\mathrm{p}}}{\mathrm{d} t}=\frac{-I_{\mathrm{p}} R_{\mathrm{p}}+V_{\text {loop }}}{L_{\mathrm{p}}} .
$$

Figure 4 shows the time-averaged values of plasma inductance, loop voltage, and plasma current as functions of the time-averaged value of $T_{\mathrm{e}}^{3 / 2}$. Because $\left\langle T_{\mathrm{e}}^{3 / 2}\right\rangle$ varies from approximately 100 to 300 among the shots, it seems that $\left\langle\eta_{\mathrm{p}}\right\rangle$ varies by approximately three times of that evaluated 


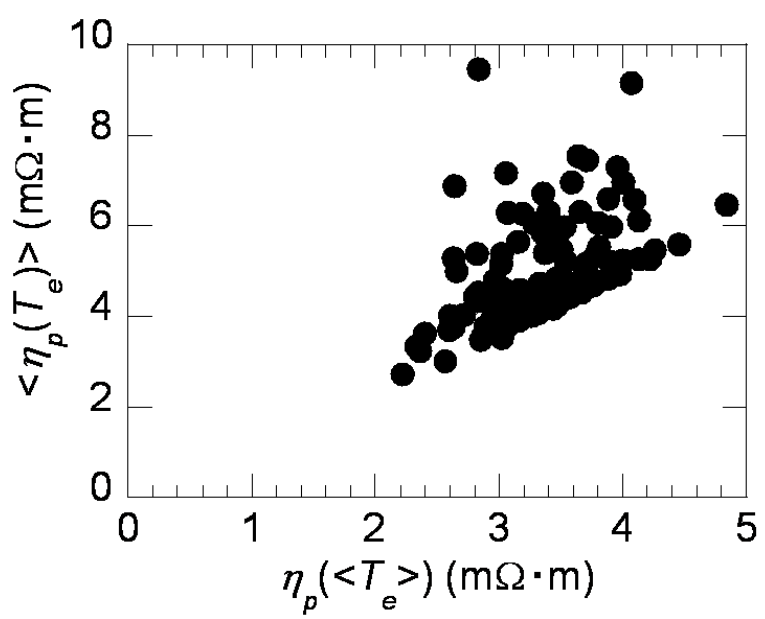

Fig. 3 Comparison between $\left\langle\eta_{\mathrm{p}}\left(T_{\mathrm{e}}\right)\right\rangle$ and $\eta_{\mathrm{p}}\left(\left\langle T_{\mathrm{e}}\right\rangle\right) .\left\langle\eta_{\mathrm{p}}\left(T_{\mathrm{e}}\right)\right\rangle$ is calculated by $\eta_{\mathrm{p}}(t)$ averaging over the hatched region in Fig. 1. $\eta_{\mathrm{p}}\left(\left\langle T_{\mathrm{e}}\right\rangle\right)$ is calculated by time-averaged $\left\langle T_{\mathrm{e}}\right\rangle$ over the hatched region in Fig. 1.

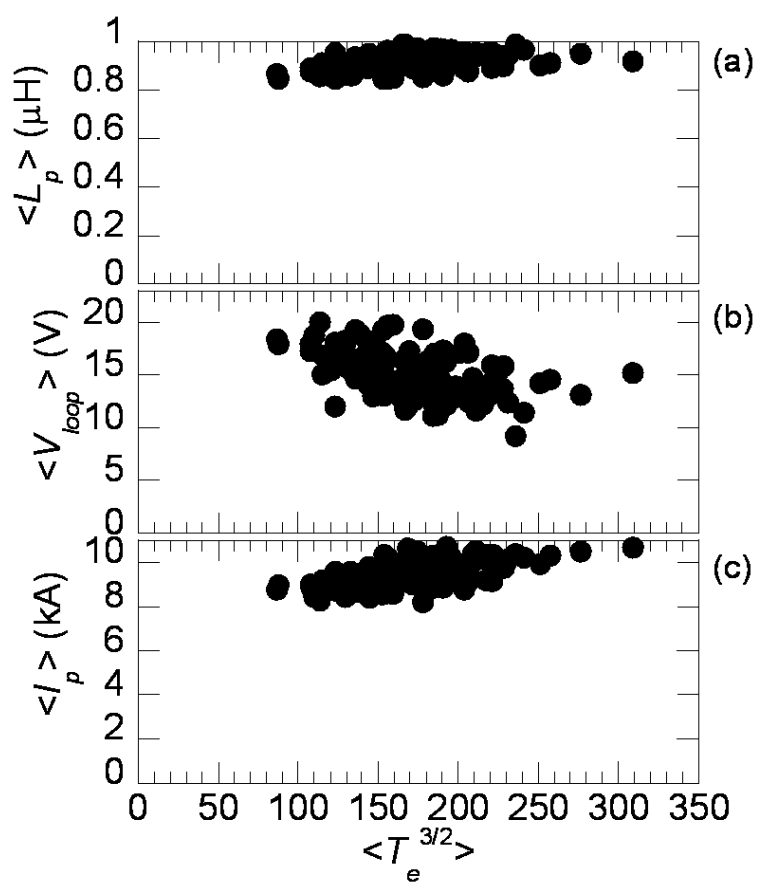

Fig. 4 Time-averaged values of (a) plasma inductance $L_{\mathrm{p}}$, (b) loop voltage $V_{\text {loop }}$, and (c) plasma current $I_{\mathrm{p}}$ during the slow decay phase, as a function of the time-averaged value of $T_{\mathrm{e}}^{3 / 2}$.

by Eq. (8). Both $\left\langle L_{\mathrm{p}}\right\rangle$ and $\left\langle I_{\mathrm{p}}\right\rangle$ slightly increase with $\left\langle T_{\mathrm{e}}^{3 / 2}\right\rangle$. On the other hand, $\left\langle V_{\text {loop }}\right\rangle$ decreases with $\left\langle T_{\mathrm{e}}^{3 / 2}\right\rangle$; however, although $\left\langle T_{\mathrm{e}}^{3 / 2}\right\rangle$ varies by approximately three times, $\left\langle V_{\text {loop }}\right\rangle$ changes only twice. We speculate that the variation of $I_{\mathrm{p}}$ decay rate is primarily determined by the change in plasma resistance among the shots from Eqs. (8) and (9).

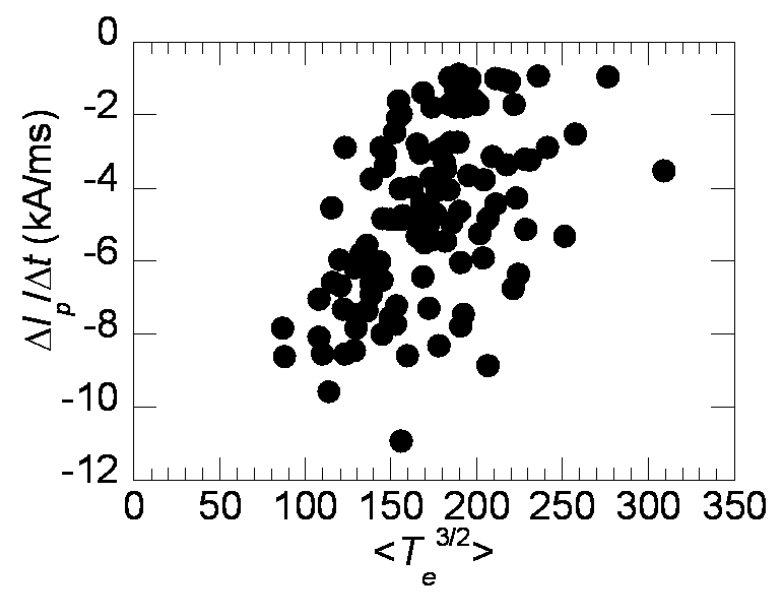

Fig. 5 Decay rate of the plasma current decay $I_{\mathrm{p}}$ during the slow decay phase as a function of $\left\langle T_{\mathrm{e}}^{3 / 2}\right\rangle$.

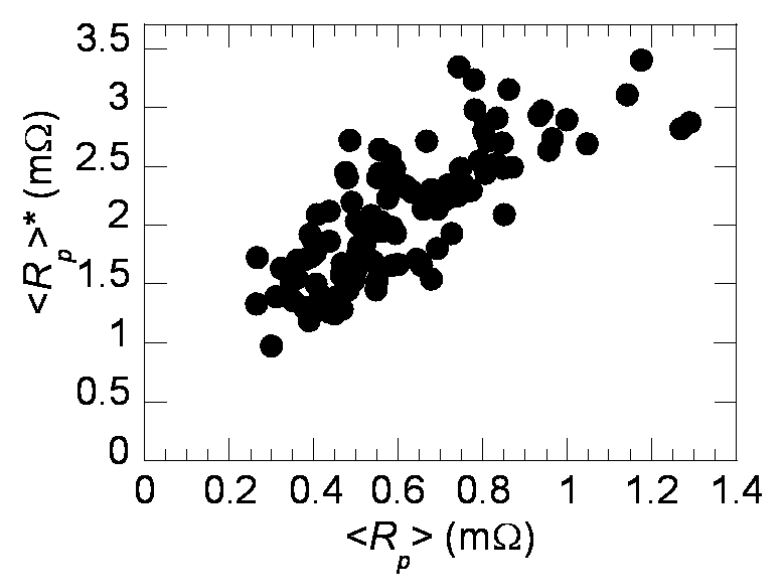

Fig. 6 Plasma resistance $\left\langle R_{\mathrm{p}}\right\rangle^{*}$ calculated from a circuit equation using experimental values of $\Delta I_{\mathrm{p}} / \Delta t,\left\langle L_{\mathrm{p}}\right\rangle,\left\langle I_{\mathrm{p}}\right\rangle$ and $\left\langle V_{\text {loop }}\right\rangle$ versus plasma resistance $\left\langle R_{\mathrm{p}}\right\rangle$ calculated by the Spitzer formula with the assumption of $Z_{\mathrm{eff}}=1$.

Figure 5 shows the rate of $I_{\mathrm{p}}$ decay $\Delta I_{\mathrm{p}} / \Delta t$ as a function of $\left\langle T_{\mathrm{e}}^{3 / 2}\right\rangle$. It is found that $\Delta I_{\mathrm{p}} / \Delta t$ increases with an increase in $\left\langle T_{\mathrm{e}}^{3 / 2}\right\rangle$ associated with the change in plasma resistance. This experimental result is in qualitative agreement with the prediction from Eq. (9); however it is not quantitatively consistent. In order to make a quantitative comparison between the experimental results and Eq. (9), we compare the plasma resistances evaluated by two calculation methods. One, which is denoted by $\left\langle R_{\mathrm{p}}\right\rangle$, is calculated from Eq. (8) using experimental values of $\left\langle T_{\mathrm{e}}^{3 / 2}\right\rangle$ and the assumption of $Z_{\text {eff }}=1$. The other, which is denoted by $\left\langle R_{\mathrm{p}}\right\rangle^{*}$, is calculated from Eq. (9) using experimental values of $\Delta I_{\mathrm{p}} / \Delta t,\left\langle L_{\mathrm{p}}\right\rangle,\left\langle I_{\mathrm{p}}\right\rangle$, and $\left\langle V_{\text {loop }}\right\rangle$. Figure 6 shows a comparison between $\left\langle R_{\mathrm{p}}\right\rangle$ and $\left\langle R_{\mathrm{p}}\right\rangle^{*}$. It is found that $\left\langle R_{\mathrm{p}}\right\rangle^{*}$ increases with $\left\langle R_{\mathrm{p}}\right\rangle$; however, $\left\langle R_{\mathrm{p}}\right\rangle^{*}$ is much larger than $\left\langle R_{\mathrm{p}}\right\rangle$, 


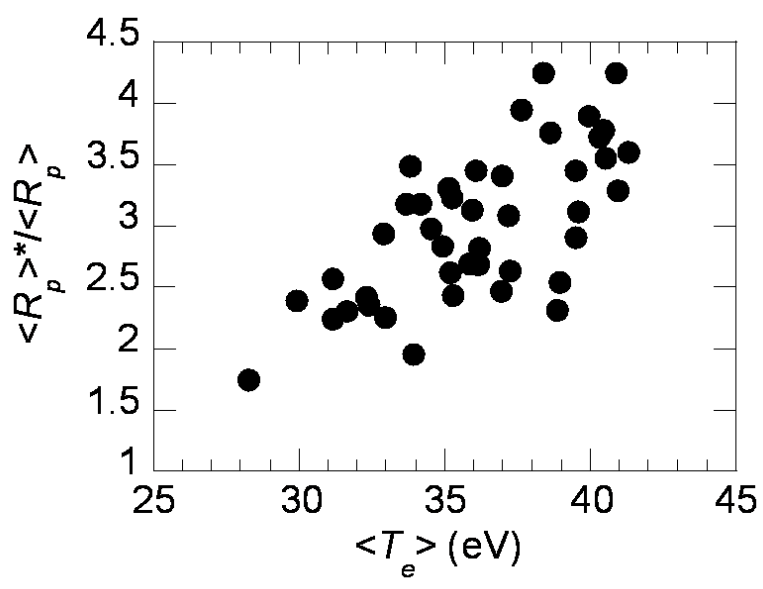

Fig. 7 Ratio between plasma resistance $\left\langle R_{\mathrm{p}}\right\rangle$ calculated by the Spitzer formula with $Z_{\mathrm{eff}}=1$, and $\left\langle R_{\mathrm{p}}\right\rangle^{*}$ calculated by a circuit equation using experimental values of $\Delta I_{\mathrm{p}} / \Delta t$, $\left\langle L_{\mathrm{p}}\right\rangle, \Delta L_{\mathrm{p}} / \Delta t,\left\langle I_{\mathrm{p}}\right\rangle$, and $\left\langle V_{\text {loop }}\right\rangle$ as a function of the timeaveraged electron temperature $\left\langle T_{\mathrm{e}}\right\rangle$.

even if the term $\Delta L_{\mathrm{p}} / \Delta t$ is considered in the plasma resistance calculation using a circuit equation. Figure 7 shows the ratios of $\left\langle R_{\mathrm{p}}\right\rangle^{*}$ to $\left\langle R_{\mathrm{p}}\right\rangle$ as a function of $\left\langle T_{\mathrm{e}}\right\rangle$. The ratio of $\left\langle R_{\mathrm{p}}\right\rangle^{*}$ to $\left\langle R_{\mathrm{p}}\right\rangle$ increases monotonically with $\left\langle T_{\mathrm{e}}\right\rangle$. This result could suggest that the effective charge $Z_{\text {eff }}$ increases with $\left\langle T_{\mathrm{e}}\right\rangle$ in the experiments, although $Z_{\mathrm{eff}}$ is assumed to be unity in our calculation. Unfortunately, there are no experimental data for $Z_{\mathrm{eff}}$ at this moment, but the discrepancy between $\left\langle R_{\mathrm{p}}\right\rangle^{*}$ and $\left\langle R_{\mathrm{p}}\right\rangle$ will be discussed in the next section.

\section{Discussion}

In our experiment, the working gas is hydrogen. The limiter and vacuum vessel are made of molybdenum and stainless steel, respectively. If the electron temperature dependence of the ratio of $\left\langle R_{\mathrm{p}}\right\rangle^{*}$ to $\left\langle R_{\mathrm{p}}\right\rangle$ in Fig. 7 is determined by the variation of $Z_{\mathrm{eff}}$, then $Z_{\mathrm{eff}}$ should vary from 2 to 4.5 in the range of electron temperature from 28 to $42 \mathrm{eV}$. With $1 \%$ iron impurity in the plasma, $Z_{\mathrm{eff}}$ could be varied from 1.25 to 1.33 at an electron temperature from 28 to $42 \mathrm{eV}$. In this analysis, we have used the ionization states of iron calculated by Arnaud and Rohtenflug [17]. In Ref. [17], the main ionization states of iron are the fifth and sixth charged states at $T_{\mathrm{e}}=28 \mathrm{eV}$, and are the sixth and seventh charged states at $T_{\mathrm{e}}=42 \mathrm{eV}$. Specific numerical values are as follows: $n_{\mathrm{Fe}+3}: n_{\mathrm{Fe}+4}$ : $n_{\mathrm{Fe}+5}: n_{\mathrm{Fe}+6}: n_{\mathrm{Fe}+7}: n_{\mathrm{Fe}+8}=0.001: 0.057: 0.43:$ $0.38: 0.12: 0.004$ at $T_{\mathrm{e}}=28 \mathrm{eV}$ and $n_{\mathrm{Fe}+4}: n_{\mathrm{Fe}+5}:$ $n_{\mathrm{Fe}+6}: n_{\mathrm{Fe}+7}: n_{\mathrm{Fe}+8}: n_{\mathrm{Fe}+9}=0.008: 0.13: 0.42: 0.35:$ $0.091: 0.001$ at $T_{\mathrm{e}}=42 \mathrm{eV}$. The assumption of $1 \%$ iron impurity in plasma is insufficient to explain the discrepancy. However, we should measure the value of $Z_{\text {eff }}$ experimentally in the future in order to form a more comprehensive discussion. Another possibility to explain the dis-

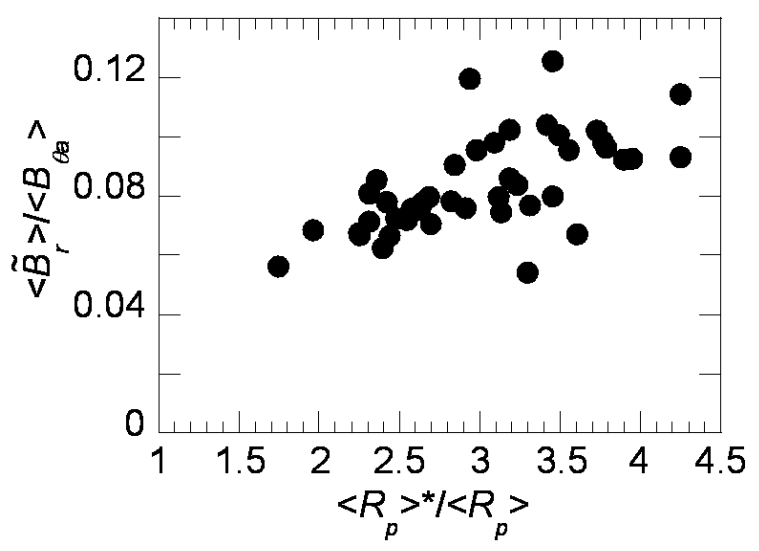

Fig. 8 Normalized amplitude of the spontaneous radial magnetic field fluctuation as a function of $\left\langle R_{\mathrm{p}}\right\rangle^{*} /\left\langle R_{p}\right\rangle$. $B_{\theta \mathrm{a}}$ is the poloidal magnetic field at the plasma surface.

crepancy is the anomalous resistivity because of magnetic fluctuations associated with magnetic field line reconnection [18]. In Ref. [18], detailed experiments on this anomalous resistivity were performed by Stenzel and Gekelman within a comparatively large facility ( $1 \mathrm{~m}$ in diameter, $2 \mathrm{~m}$ long). A rarefied plasma was produced at plasma density of $10^{12} \mathrm{~cm}^{-3}$ with an electron temperature of approximately $10 \mathrm{eV}$. They reported the increase of the plasma resistivity by two orders of magnitude because of anomalous electron scattering. Figure 8 shows the normalized amplitude of magnetic fluctuation of the internal radial magnetic field $B_{\mathrm{r}}$ as a function of the ratio of $\left\langle R_{\mathrm{p}}\right\rangle^{*}$ and $\left\langle R_{\mathrm{p}}\right\rangle$. It is found that the amplitude of internal magnetic fluctuation increases with this ratio. This could suggest that the plasma resisitivity may be influenced by anomalous resistivity induced by magnetic fluctuations associated with MHD instability.

\section{Summary}

We have verified the current decay model to establish a database of the current decay time using the slow decay phase of HYBTOK-II disruptive discharges. It is experimentally confirmed that the decay rate of the plasma current during the current quench becomes quicker with an increase in the plasma resistance, which is consistent with the current decay model. The discrepancy in plasma resistivities, estimated from the current decay model and calculated by the classical Spitzer formula, is discussed based on the effective charge in plasma and anomalous resistivity associated with magnetic perturbation. In order to evaluate the model more accurately in the future, direct measurement of the effective charge in the plasma is necessary.

\section{Acknowledgments}

We acknowledge the technical support provided by Mr M. Takagi in Nagoya University. This work was par- 
tially supported by the Grant-in-Aid for Scientific Research from the Japan Society for the Promotion of Science (JSPS) Research Fellowships for Young Scientists (No. 198456).

[1] R.O. Sayer, Y.K.M. Peng, S.C. Jardin, A.G. Kellman and J.C. Wesley, Nucl. Fusion 33, 969 (1993).

[2] K. Masaki, T. Ando, K. Kodama, T. Arai et al., J. Nucl. Mater. 220-222, 390 (1995).

[3] Progress in the ITER Physical Basis, Nucl. Fusion 47, S171 (2007).

[4] H. Ohwaki, M. Sugihara and A. Hatayama, Plasma Fusion Res. 1, 016 (2006).

[5] M. Bakhtiari and D.G. Whyte, Phys. Plasmas 13, 112511 (2006).

[6] D.C. Robinson and K.M. McGuire, Nucl. Fusion 19, 115 (1979).

[7] K.M. McGuire and D.C. Robinson, Phys. Rev. Lett. 44, 1666 (1980).
[8] I.H. Hutchinson, Phys. Rev. Lett. 37, 338 (1976).

[9] A.H. Morton, Nucl. Fusion 16, 571 (1976).

[10] A. Vannucci, I.C. Nascimento and I.L. Caldas, Plasma Phys. Control. Fusion 31, 147 (1989).

[11] L. Giannone, R.C. Cross and I.H. Hutchinson, Nucl. Fusion 27, 2085 (1987).

[12] S. Takamura, Y. Kikuchi, Y. Uesugi and M. Kobayashi, Nucl. Fusion 43, 393 (2003).

[13] L. Spitzer and R. Härm, Phys. Rev. 89, 977 (1953).

[14] M. Okamoto, T. Yamada, T. Hiraishi, Y. Kikuchi, N. Ohno and S. Takamura, J. Nucl. Mater. 363-365, 1076 (2007).

[15] M. Okamoto, S. Takamura, N. Ohno, S. Kajita, Y. Kikuchi, Y. Uesugi, T. Ozeki, Y. Kawano and M. Sugihara, Nucl. Fusion 47, 1106 (2007).

[16] J.A. Wesson, Tokamaks 3rd edition (New York: Oxford University Press, 2004) chapter 3.

[17] M. Arnaud and R. Rohtenflug, Astron. Astrophys. Suppl. Ser. 60, 425 (1985).

[18] N. Wild, W. Gekelman and R.L. Stenzel, Phys. Rev. Lett. 46, 339 (1981). 